\title{
La Place de la Traduction Automatique dans l'Enseignement de la Traduction
}

Erdinç ASLAN ${ }^{1}$

\section{Résumé}

Les développements rapides de la traduction automatique ces dernières années ont considérablement affecté le domaine de la traduction. Les nouvelles méthodes qui sont appliquées ont fourni des améliorations significatives dans la qualité de la traduction. D'une part cette situation a fait de la traduction automatique d'un domaine sur lequel les grandes entreprises se concentrent et réalisent des investissements importants, et par conséquent, elle a augmenté la part de marché de la traduction automatique dans le secteur, et d'autre part elle l'a rendu populaire en permettant aux gens de s'intéresser à la traduction automatique. Malgré cela, la mesure dans laquelle ces développements peuvent être reflétés dans le programme d'enseignement de la traduction est discutée par les enseignants en traduction. Cette étude se concentre sur la place de la traduction automatique dans l'enseignement de la traduction et examine comment ces systèmes peuvent être reflétés dans le programme d'enseignement de la traduction.

\author{
Mots-clés \\ traduction automatique \\ enseignement de la traduction \\ traduction \\ technologies de traduction
}

\section{À Propos de l'Article}

Arrivée: $\quad 29.05 .2021$

Acceptation: 18.07.2021

Doi:

10.20304/humanitas.944629

\section{Makine Çevirisinin Çeviri Eğitimindeki Yeri}

$\ddot{O} z$

Makine çevirisinde son yıllarda yaşanan hızlı gelişmeler çeviri alanını önemli ölçüde etkilemiştir. $\mathrm{Bu}$ alanda uygulanan yeni yöntemler çeviri kalitesinde dikkate değer biçimde iyileşmeler sağlamıştır. Bu durum bir yandan makine çevirisini büyük şirketlerin üzerinde durduğu ve önemli yatırımlar yaptığı bir alan haline getirmiş ve bunun sonucu olarak makine çevirisinin sektördeki pazar payını artırmış diğer yandan ise sıradan insanların da makine çevirisine ilgi duymasına olanak sağlayarak kullanımını yaygınlaştırmıştır. Fakat bunlara rağmen bu gelişmelerin çeviri eğitimi müfredatına ne kadar yansıtılabildiği çeviri eğitimcileri tarafından tartışılmaktadır. Bu çalışmada makine çevirisinin çeviri eğitimindeki yeri üzerinde durulmakta ve bu sistemlerin çeviri eğitimi müfredatına nasıl yansıtılabileceği ele alınmaktadır.
Anahtar Kelimeler

makine çevirisi

çeviri eğitimi

çeviri

çeviri teknolojileri

\section{Makale Hakkında}

Geliş Tarihi: 29.05.2021

Kabul Tarihi: 18.07.2021

Doi:

10.20304/humanitas.944629

\footnotetext{
${ }^{1}$ Maître de conférences, Université Marmara, Faculte des Arts et des Sciences, Departement de Traduction et d'interpretation, Istanbul/Turquie, erdinc.aslan@marmara.edu.tr, ORCID: 0000-0002-8484-1450
} 


\section{Introduction}

Dans le passé, l'activité de traduction qu'on fait utilisant du papier, un stylo et des dictionnaires imprimés se fait maintenant principalement dans l'environnement informatique avec la technologie en développement. L'une des applications les plus importantes offertes par les technologies informatiques au domaine de la traduction est la traduction automatique. La traduction automatique a subi de grands changements et transformations dans les dernières années. L'interaction homme-machine est plus avancée que jamais. Les nouvelles méthodes appliquées par des chercheurs, l'augmentation de la quantité de données, les développements des technologies informatiques et Internet ont apporté une contribution extrêmement importante au développement de la traduction automatique et des améliorations significatives dans la qualité de la traduction. Cependant, la traduction automatique est devenue un domaine sur lequel les grandes entreprises se concentrent et réalisent des investissements importants. En conséquence, la part de marché de la traduction automatique dans le secteur a considérablement augmenté ces dernières années et continue d'augmenter de jour en jour. Parce qu'avec les nouvelles méthodes appliquées, la confiance dans la qualité de la traduction automatique a augmenté, par rapport à celle accordée aux anciennes. Elle est désormais privilégiée car elle est plus rapide et moins chère.

D'autre part, les systèmes de langage verbal en temps réel, l'introduction de différentes applications de traduction appelées La Traduction d'Images ou La Traduction Visuelle (Picture Translation ou Visual Translation), qui traduisent des textes écrits dans des images telles que des photographies, ont rendu la traduction automatique plus populaire. Les gens ont commencé à utiliser ces applications pour produire des solutions pratiques dans le processus de la traduction. Les smartphones, qui se sont transformés en ordinateurs dotés de nouveaux systèmes d'exploitation et se sont généralisés en peu de temps, jouent un grand rôle dans l'émergence de cette situation. Avec les applications gratuites proposées sur les smartphones, la traduction automatique a atteint un public beaucoup plus large et est devenue un domaine d'intérêt non seulement pour les scientifiques et les traducteurs travaillant dans ce domaine, mais aussi d'autres personnes ayant besoin de la traduction

Les réseaux sociaux constituent un autre facteur de l'intérêt croissant pour la traduction automatique. Car le nombre d'utilisateurs des réseaux sociaux augmente de jour en jour. Les utilisateurs de différents pays du monde qui se connectent aux réseaux sociaux communiquent entre eux via ces plateformes. Les sites des réseaux sociaux qui fournissent des services intégrés aux systèmes de la traduction automatique, offrent aux utilisateurs la possibilité de 
visualiser la traduction d'un article ou d'un commentaire rédigé dans une autre langue en cliquant sur le lien « Voir l'original » au bas de cet article ou commentaire. Les réseaux sociaux qui fournissent des services vidéo, proposent aussi des services de traduction de soustitres au public qui ne parle pas la même langue que la langue de la vidéo, en utilisant la traduction automatique. En outre, les réseaux sociaux sont une source importante de données pour la traduction automatique. Et en ce qui concerne la traduction automatique, ces données sont très primordiales. Car la production de bons résultats dépend de leur quantité. À mesure que la quantité de données augmente, le taux d'obtention de résultats positifs augmente aussi. Les partages réalisés via les sites de réseaux sociaux sont collectés en temps réel et utilisés comme données pour les systèmes de la traduction automatique.

Sur la traduction automatique, on constate un développement remarquable ces dernières années : c'est le cloud computing. Cloud computing systems, qui permet de localiser le logiciel sur un serveur Internet sans être installé sur un ordinateur et d'accéder à ces serveurs avec un appareil connecté à Internet, permet aux mémoires de la traduction automatique. Lors de l'utilisation de la mémoire de traduction, des textes préalablement traduits à l'aide de la traduction automatique sont à nouveau analysés avec les modèles grammaticaux et les usages courants de la langue cible et les meilleurs résultats sont essayés pour être produits. Au cours de ce processus, le moteur de la traduction automatique est constamment mis à jour avec des exemples de traduction humaine.

En outre, il est prévu que l'intérêt pour la traduction automatique augmentera encore plus dans les années à venir. Il y a plusieurs raisons à cela. La première est que, comme mentionné ci-dessus, elle offre des solutions pratiques aux besoins de traduction des gens, et elle est facile à utiliser et gratuite avec des appareils tels que les smartphones et les tablettes. La seconde est que les entreprises préfèrent la traduction automatique car elle est plus rapide et plus économique. Ainsi, les investissements dans la traduction automatique devraient augmenter dans les années à venir. Dans une étude menée par STATISTA Research Company (2019), on estime que la part du marché mondial de la traduction automatique, qui était de 630 millions USD en 2019, passera à 1500 millions USD en 2024.

Sans aucun doute, tous ces développements dans le domaine de la traduction automatique engendrent des résultats significatifs pour l'enseignement de la traduction. L'enseignement de la traduction, qui est une sous-branche de la traductologie appliquée est au centre de ces développements. Il est incontestable que la technologie fait désormais partie intégrante de la traduction. Car aujourd'hui, le processus de traduction se déroule en grande 
partie dans des environnements technologiques et profiter des opportunités offertes par la technologie est devenu une nécessité plutôt qu'un choix. Tous les processus tels que la communication avec les clients, la promotion, la publicité, le marketing, la traduction, l'évaluation, la correction, la livraison et le paiement sont en grande partie réalisés dans des environnements technologiques. Pour cette raison, l'un des principaux objectifs de l'enseignement de la traduction, c'est de garantir que les candidats traducteurs acquièrent les compétences nécessaires pour mener à bien tous ces processus. Cependant, on discute actuellement de la mesure dans laquelle ces développements peuvent être reflétés dans le programme de l'enseignement de la traduction. Il faut souligner que la traduction automatique était généralement gérée par des ingénieurs informaticiens jusqu'à récemment, et qu'elle était notamment analysée en termes de logiciels. Dans cette étude on se concentre sur la place de la traduction automatique dans l'enseignement de la traduction et examine comment ces systèmes peuvent être reflétés dans le programme d'enseignement de la traduction et comment la traduction automatique peut être utilisée de manière plus efficace et efficiente. A cet effet, on essaie de concrétiser les informations en faisant des évaluations sur les études antérieures. En outre pendant ce travail, la traduction en français des références ou des citations appartiennent aux théoriciens étrangers est faite par nous.

\section{L'enseignement de la traduction et La traduction automatique}

L'enseignement de la traduction, qui est le domaine d'application des études de traduction, vise à étudier des personnes ayant la qualité souhaitée, comme dans tous les autres domaines de l'enseignement. Pour atteindre cet objectif, les étudiants doivent être étudiés de manière polyvalente. En fait, avec le développement de la technologie et la quantité croissante d'informations, les sujets sur lesquels se concentre l'enseignement de la traduction se diversifient également. Lorsqu'on parle de l'enseignement de la traduction, il est nécessaire de se concentrer sur le concept de compétence de traduction. Car l'un des objectifs les plus importants de l'enseignement de la traduction, c'est de faire acquérir des compétences de traduction aux étudiants. Le concept de compétence de traduction peut être simplement défini comme la capacité de traduire. La compétence de traduction est essentiellement un concept supérieur ou général qui se compose de diverses sous-compétences. Ce concept peut être mis à jour selon les conditions et les besoins de l'époque ou peut être élargi, et ses limites redéfinies. Les objectifs de l'éducation peuvent être mis à jour en fonction des conditions de l'âge, les besoins de l'individu et de la société, et par conséquent, les domaines de compétence que le fournisseur de traduction doit couvrir, qui font partie des objectifs de l'enseignement de 
la traduction, peuvent être remodelés en fonction des temps, des conditions et des besoins. En effet, si ces sous-compétences, auparavant classées de différentes manières par différents scientifiques (Bell 1991, Kautz 2000, Pym 2003, Hurtado 1996), n'étaient pas incluses dans l'acquisition de technologies, les théoriciens considèrent aujourd'hui (Pym 2013, Eser 2014, Yazar 2014, Durukan ve Çelikay 2018) le fournisseur de technologie comme un subordonné du fournisseur de traduction.

La compétence technologique dans la traduction fait référence à la capacité d'utilisation des outils technologiques dans le processus de traduction, en d'autres termes, les technologies de traduction de manière efficace et efficiente. Yazar (2014) soutient qu'aujourd'hui, une personne qui ne connaît pas les logiciels AutoCAD ne peut pas être ingénieur, et qu'une personne qui ne connaît pas les technologies de traduction, c'est-à-dire les programmes de traduction assistée par ordinateur (TAO), ne peut pas être traducteur ou traductrice. De plus, Haldan (2018) déclare que la reconnaissance de la langue, le développement de la terminologie, la connaissance d'un large éventail d'outils multimédias tels que la configuration, l'utilisation et la maintenance de bases de données pour créer la terminologie et le suivi des développements dans le domaine des langues, des technologies de l'information et de la communication sont également inclus à la compétence technologique, hors de la connaissance de l'utilisation pour l'écriture informatique et la recherche.

En ce qui concerne les technologies de traduction, l'une des premières applications qui vient à l'esprit, c'est la traduction automatique. La traduction automatique est la traduction faite automatiquement d'une langue à une autre à l'aide d'un logiciel informatique sans intervention humaine. Avec l'amélioration continue de la qualité de la traduction, la traduction automatique retient plus encore l'attention de jour en jour et il est impensable de la laisser à l'écart du programme d'enseignement de la traduction. En fait, Austermuehl (2013) affirme que le succès de la traduction automatique statistique et l'augmentation des capacités de traduction ont changé la nature de la traduction et avec ce changement, la nécessité de restructurer le programme de formation est apparue. Yuste, (2001) déclare que la traduction automatique occupe une place très importante de nos jours et souligne que l'enseignement de la traduction automatique doit être avant tout pratique et réaliste, ainsi que centré sur l'étudiant.

Malgré cela, on voit que la traduction automatique n'est pas suffisamment incluse dans le programme d'enseignement de la traduction et, comme mentionné ci-dessus, cette question a été généralement traitée par des ingénieurs informaticiens et a été étudiée surtout en termes 
de logiciels. Alkan (2013) souligne qu'il est important pour les établissements de prendre en compte les conditions actuelles du marché de la traduction tout en organisant leurs programmes, mais qu'il n'y a pas de consensus dans les études de traduction sur la manière de l'inclure dans l'enseignement de la traduction, qui serait également valable pour la traduction automatique. Selon Ersoy et Balkul (2012) l'accent n'est pas suffisamment mis sur les systèmes de traduction automatique et les autres technologies de traduction dans les départements de traduction, parce que les universités sont sous-financées et que le soutien à l'achat de matériels et d'équipements pour les études expérimentales dans les domaines de la science n'est pas fourni aux études de traduction comme dans les autres domaines des sciences sociales.

Austermuehl (2013) résume la couverture insuffisante de la traduction automatique dans le programme de l'enseignement de la traduction.

- Les solutions technologiques telles que les mémoires de traduction ou les systèmes de la traduction automatique statistique sont développées soit en dehors des universités, c'est-à-dire dans des entreprises de traduction ou des entreprises ayant des besoins de traduction, soit dans les universités qui n'ont pas de département de traduction.

Il n'y a pas suffisamment de recherche, en particulier de recherche empirique, sur ces outils, leur impact sur les processus de traduction, les produits et les producteurs, et donc le plus souvent pas de base solide pour développer des cours.

De nombreux traducteurs formateurs n'ont pas l'expertise professionnelle et / ou technologique pour mettre en œuvre rapidement et correctement les technologies de traduction dans leur enseignement.

- Il y a tellement d'autres choses à enseigner, et souvent même pas assez de temps pour cela.

Malgré tout cela, les développements dans le domaine de la traduction automatique et l'intérêt des gens pour la traduction automatique ont sensibilisé les enseignants en traduction et la place de la traduction automatique dans l'enseignement de la traduction a commencé à être remise en question. Des chercheurs tels que Bulut (2019), Killman (2015), Rossi (2017) y ont contribué en se concentrant sur l'intégration de la traduction automatique dans l'enseignement de la traduction. Dans ces études, des sujets tels que la composition du contenu, l'inclusion de nouvelles approches dans le programme, les méthodes à appliquer, l'évaluation du point de vue des étudiants sur la traduction automatique, l'analyse des erreurs, 
l'interaction homme-machine et les approches de la traduction automatique, sont examinés de manière empirique et descriptive.

Ces changements et cette transformation rapides de la traduction automatique ont également soulevé le débat sur la manière dont le programme devrait être conçu. Quand nous regardons les développements de ces dernières années, il y a eu des changements dans le domaine de la traduction automatique à une vitesse vertigineuse. De nouvelles méthodes telles que la traduction neuronale, la quantité croissante de données et les améliorations évidentes de la qualité de la traduction montrent que ce changement se poursuivra au même rythme dans les périodes à venir. Ces évolutions révèlent sans aucun doute la nécessité de changer et d'actualiser constamment le programme d'enseignement.

Cependant, si un programme basé sur les applications en usage aujourd'hui est réalisé, il sera obsolète lorsque de nouvelles applications émergeront, et un programme basé sur de nouveaux développements et de nouvelles approches devront être présentées aux étudiants. Pour cette raison, il serait plus approprié de présenter un programme qui comprend des sujets tels que la compréhension de la logique de fonctionnement de ces systèmes dans les cours, la sensibilisation aux applications nouvelles et différentes, et le développement des compétences de résolution de problèmes face aux limites des nouvelles technologies, à la fois en termes des technologies de la traduction automatique et de traduction en général.

\section{Le contenu du cours de la traduction automatique}

Aujourd'hui, bien que la traduction automatique ne soit pas incluse dans le programme de l'enseignement de la traduction au niveau souhaité, des cours de traduction automatique sont organisés dans diverses universités qui offrent une formation de traduction. Si ces cours se déroulent selon des programmes préparés à l'initiative des professeurs qui assurent les cours, on constate qu'il n'y a généralement pas de consensus sur la manière d'intégrer la traduction automatique dans l'enseignement de la traduction. Alors que l'idée de faire une traduction avec la traduction automatique prend du poids, diverses hypothèses sont avancées sur des points théoriques et pratiques, et peuvent être ici abordées. Conformément à ces idées, des cours pratiques sont également mis en œuvre dans diverses universités.

L'un des problèmes les plus marquants de la traduction automatique est la post-édition. La traduction automatique ayant certaines limites, la sortie doit être prête à être utilisée. Les limites de la traduction automatique sont basées sur des facteurs comme le fait que les langues naturelles ont des rôles communicatifs différents les uns des autres, qu'elles ont des structures communes similaires mais ont également des structures différentes. Les limites syntaxiques, 
sémantiques, idiomatiques, lexicales etc., posent certaines difficultés à ceux qui utilisent la traduction automatique. La manière de résoudre ces difficultés et les stratégies qui seront utilisées pour rendre le résultat prêt à l'emploi sont mises en œuvre dans la phase de la postédition. Par conséquent, la post-édition dans la traduction automatique devient de plus en plus importante, et cela a commencé à changer le rôle des traducteurs dans le processus de traduction.

Dans le monde d'aujourd'hui, où les méthodes traductives se changent, les traducteurs travaillent comme des éditeurs en effectuant des processus de pré-édition et de post-édition afin d'améliorer la qualité du texte cible. En fait, les entreprises qui exigent des services de traduction paient pour le service, moins cher, de la pré-édition et de la post-édition, préférant la traduction automatique au traducteur humain plus cher. Il convient de souligner que cette situation est aussi rapide que bon marché et que c'est une méthode privilégiée pour les besoins urgents de traduction. En effet, on obtient alors un texte presque prêt à l'emploi produit par la machine lors du processus de la post-édition. Le traducteur augmente la qualité de la sortie en prenant les dispositions nécessaires sur ce texte et prépare le texte à l'utilisation. La préédition du texte est également nécessaire pour réduire le taux d'erreur à la sortie de la traduction parce que les entrées incorrectes entraîneront une production erronée.

En outre, divers logiciels sur l'édition de la traduction automatique ont également été lancés, et ce processus peut désormais s'exécuter automatiquement. Ce système, appelé postédition automatique, vise à corriger les erreurs systématiques et répétitives dans la sortie de la traduction automatique. Vu et Haffari (2018) soutiennent que les travaux antérieurs sur la post-édition automatique se sont concentrés spécifiquement sur la mise en forme de la postédition automatique, mais que son système a créé des problèmes tels que des corrections excessivement inutiles et une qualité de traduction réduite. Negri et d'autres (2017) indiquent que certaines études pour la post-édition automatisée se concentrent sur les modèles neuronaux en ligne et préconisent d'utiliser les commentaires des traducteurs humains plutôt que de former des réseaux à partir de zéro, puisque les corrections apportées par les traducteurs humains sont une source inestimable d'informations à utiliser pour améliorer les systèmes.

Que ce soit à la main ou à l'aide d'un logiciel, l'édition de la traduction automatique fait aussi l'objet de l'enseignement de la traduction en tant que compétence éditoriale et fait partie des compétences que les étudiants devraient acquérir. Par conséquent, le programme devrait inclure cette étude dans le cadre des cours de traduction automatique. Malgré le 
manque d'experts, la nécessité d'une coopération interdisciplinaire et le manque d'équipements techniques, on constate que cette étude, qui n'a pas encore été abordée au niveau souhaité dans l'enseignement de la traduction, sera inévitablement incluse dans le programme. Car, on constate que les études sur ce sujet augmentent de jour en jour et que l'intérêt augmente.

Cependant, il convient de souligner que des cours de traduction automatique et de post-édition ont commencé à prendre place dans les départements dispensant un enseignement de traduction. Dans un cours dirigé par Koponen (2015) à l'Université d'Helsinki à l'automne 2014, entièrement axé sur la traduction automatique et la post-édition, le contexte théorique et les exercices pratiques de la traduction automatique et de post-édition ont été abordés. Les sujets abordés dans le cours sont énumérés ci-dessous :

- La théorie et l'histoire de la traduction automatique et la post-édition

- Utilisation pratique de la traduction automatique et de la post-édition

- $\quad$ Langage contrôlé et pré-édition pour la traduction automatique

- $\quad$ Post-édition sans texte source

- $\quad$ Recherche de processus post-édition

- $\quad$ Niveaux de qualité et directives de la post-édition

- Évaluation de la qualité de la traduction automatique et études de la postédition

- Compétences sur la post-édition

Şahin (2014) a sollicité les étudiants du département de traduction et d'interprétation avec des domaines spéciaux de la traduction automatique dans le cadre des dernières questions de la post-édition en traduction dans une université de fondation en Turquie. Dans ce cours, il présente les systèmes de la traduction automatique pour des étudiants et les sensibilise à leur place dans la traduction, et donne aussi des informations aux étudiants sur la post-édition. Diverses activités liées à la traduction automatique et à la post-édition ont été menées dans ce cours avec des textes de différents domaines tels que le droit, la technologie et les médias, et elles se sont déroulées dans le laboratoire informatique pendant cinq semaines, trois heures par semaine. À la fin du cours, la majorité des étudiants ont déclaré que leurs compétences de traduction se sont améliorées davantage que dans les cours de traduction classiques sans la traduction automatique. 
Un autre cours sur l'intégration de la traduction automatique dans l'enseignement de la traduction a été mené par Bulut (2019) à l'Université Arel d'Istanbul. Pendant le cours organisé au semestre d'automne de l'année universitaire 2018-2019, les étudiants devaient d'abord traduire eux-mêmes trois textes de l'anglais vers le turc, puis ils ont été invités à interpréter la sortie de la traduction automatique en traduisant d'abord les trois textes de l'anglais vers le turc par eux-mêmes puis en les traduisant via la traduction automatique, et à comparer la sortie de la traduction humaine avec la sortie de la traduction automatique. Deux semaines sont accordées pour l'étude de chaque texte source. Au cours de la première semaine, les étudiants ont été invités à venir en classe en ayant traduit le texte source et commenté les processus d'analyse et de recherche de texte axés sur la traduction, pour expliquer comment ils ont analysé le texte source, et en faire découler une discussion en classe sur l'analyse et sur la traduction. La semaine suivante, les étudiants ont été invités à venir en classe en traduisant le texte source avec le système de traduction automatique de leur choix, écrivant cette fois un commentaire sur leurs observations concernant le résultat brut de la traduction automatique. Bien que les étudiants aient démontré des perceptions positives et négatives sur la traduction automatique dans leur analyse, ils ont personnalisé la machine en faisant une comparaison entre le traducteur humain et la machine à traduire, qu'ils ont définis comme « un partenaire et un concurrent pour coopérer».

D'autre part, dans l'enseignement de la traduction, on constate que le cours sur les technologies de la traduction se concentre davantage sur la mémoire de traduction. Car les mémoires de traduction sont les outils indispensables pour les traducteurs. Aujourd'hui, il n'y a pratiquement pas de traducteur qui ne consulte pas les mémoires de traduction. Par conséquent, il est inévitable que ces outils fassent l'objet d'un cours sur les technologies de traduction. De plus, la capacité de ces outils est augmentée en utilisant les mémoires de traduction de manière intégrée avec la traduction automatique. Les mémoires de traduction alimentent la base de données de la traduction automatique, contribuant à des traductions plus précises. Il y a donc une interaction importante entre les deux outils. Pym (2013) attire l'attention sur cette situation et déclare que plus ces outils sont utilisés, meilleurs ils seront, et que plus ils sont en ligne, plus les utilisateurs y auront accès via le cloud et les bases de données d'autres personnes.

Grâce aux technologies cloud, de plus en plus importantes aujourd'hui, il est devenu plus facile d'intégrer la traduction automatique aux mémoires de traduction et de mettre en œuvre ces applications dans l'enseignement de la traduction. Car sans avoir besoin 
d'installation, ces systèmes ne peuvent être utilisés à partir de tous les ordinateurs en ligne qu'en achetant un abonnement. La commodité la plus frappante de ces systèmes est qu'il n'y a pas de problème de mise à jour. Dans les applications de bureau, le logiciel devait être mis à jour presque chaque année pour un certain montant. Comme cela créait un coût pour les établissements de l'enseignement de la traduction, la plupart des établissements ne pouvaient pas constamment actualiser ces logiciels.

L'intégration de la traduction automatique dans les mémoires de traduction est l'un des sujets à aborder dans les cours de la traduction automatique. En effet, d'une part, les étudiants amélioreront leurs compétences telles que l'utilisation de différents types d'outils technologiques, l'adaptation aux nouvelles technologies et l'apprentissage par eux-mêmes, d'autre part, cette situation créera une prise de conscience du changement de paradigme dans le marché de la traduction.

\section{La traduction automatique et les approches pédagogiques}

Lorsqu'on examine les travails, on constate que les approches liées à l'enseignement de la traduction automatique sont discutées dans le cadre des pédagogies de l'enseignement des technologies de traduction. Ces approches se divisent généralement en deux. La première peut être considérée comme controversée, la seconde comme relevant d'une conception consumériste. Selon Alkan (2013) qui parle de ces deux approches différentes, la première vision consiste à planifier l'éducation technologique en fonction des objectifs de fournir des compétences de base telles que la résolution de problèmes, le questionnement, l'autoapprentissage et le travail d'équipe, et de contribuer à sensibiliser aux conditions actuelles du marché. Le deuxième point de vue consiste, lui, à développer une approche axée sur le marché dans l'enseignement de la traduction, car le but de la formation est de former des traducteurs avec les qualifications requises sur le marché, et c'est une approche consumériste.

Deux catégories différentes sont mentionnées comme des approches minimalistes et maximalistes. Selon Balkul (2015), les universités qui offrent une formation sur la traduction à notre époque où les développements technologiques progressent rapidement, adoptent une approche minimaliste au lieu d'enseigner aux étudiants les technologies de traduction largement utilisées dans l'industrie de la traduction d'aujourd'hui. De manière générale, elle vise à montrer la logique de fonctionnement de ces programmes aux étudiants et à faire acquérir la capacité de faire face aux tâches du traducteur qui doivent être préparées avec différents outils de traduction assistée par ordinateur. Dans l'approche maximaliste, l'objectif, c'est que les étudiants qui disposent d'une formation sur la traduction au sens général 
interagissent autant que possible avec les outils de traduction qu'ils peuvent rencontrer dans leur vie professionnelle.

De ce point de vue, basé sur l'approche contentieuse de l'enseignement de la traduction automatique, un cursus visant à sensibiliser à la traduction automatique, à la résolution de problèmes et au questionnement, à l'auto-apprentissage et au travail en collaboration peut être proposé aux étudiants. Sur la base de l'approche consumériste, un programme peut être suivi en démontrant quelles sont les compétences minimales que les étudiants doivent acquérir en matière de traduction automatique. Par exemple, dans la traduction automatique, des sujets tels que la pré-édition, la post-édition, la gestion de la terminologie, l'évaluation de la traduction automatique peuvent être déterminés comme des compétences minimales et les étudiants peuvent acquérir la capacité de gérer ces processus efficacement.

Une autre approche à considérer est l'approche constructiviste sociale. Le constructivisme social est basé sur l'établissement d'un lien entre les activités organisées culturellement ou socialement et la structuration de l'information, c'est-à-dire que la connaissance se forme par la décision commune du groupe social et que le sens se construit alors dans le contexte social des individus. En partageant ce sens qu'ils créent, ils affectent les pensées des autres individus et ils sont également affectés par ces individus (Akyol et Fer 2010, s. 882). Kiraly est l'un des scientifiques qui soutiennent que l'approche constructiviste sociale devrait être utilisée dans l'enseignement de la traduction. Les enseignants devraient transférer leurs compétences de base en matière d'acquisition d'outils au premier stade, puis se retirer progressivement, offrant aux étudiants la possibilité de développer leurs propres compétences informatiques en menant leurs études de traduction, en traitant de vrais problèmes de traduction, en corrigeant et améliorant leurs traductions pour atteindre les normes professionnelles. Les étudiants acquièrent les compétences nécessaires pour utiliser différents types de stratégies en générant des solutions raisonnables grâce à un travail de groupe collaboratif (Kiraly, 2014).

Utiliser une approche constructiviste sociale dans l'enseignement de la traduction peut aider à sensibiliser les étudiants aux concepts d'estime de soi professionnelle en introduisant les principes de la concurrence et de la coopération homme-machine. En effet, une application d'apprentissage conçue par Bulut (2019) pour intégrer la traduction automatique dans la formation des traducteurs a été mise en œuvre et les mécanismes de l'apprentissage tout au long de la vie ont été pris en compte afin d'apprendre à apprendre et à faire face aux 
difficultés imprévues que les étudiants rencontreront à l'avenir. Pym (2013) met l'accent sur le concept de l'enseignement de l'apprentissage et déclare que ce concept peut inclure :

- Capacité d'apprendre rapidement en trouvant et traitant des ressources en ligne ;

- Capacité d'évaluer l'adéquation d'un outil aux besoins techniques et au prix ;

- Capacité à étudier avec des pairs pour résoudre des problèmes d'apprentissage ;

- Capacité à évaluer de manière critique l'étude avec outils technologiques

Le concept d'enseigner l'apprentissage joue un rôle important dans les technologies de la traduction automatique et de traduction générale. Car l'enseignement de la traduction est donné dans un certain laps de temps, la technologie se développe et elle est constamment renouvelée de jour en jour. Comme dans de nombreux domaines, les technologies utilisées dans le domaine de la traduction évoluent également et créent des changements dans la réception de la traduction. La capacité des étudiants pour suivre et pour tirer bénéfice des nouveaux développements technologiques tout au long de leur vie professionnelle est directement liée à la capacité d'enseigner l'apprentissage.

\section{Conclusion}

Le changement de paradigme dans la traduction automatique se reflète également dans l'enseignement de la traduction et révèle la nécessité de mettre à jour le programme pour inclure à nouveau ces changements. En fait, la compétence de traduction que l'enseignement de la traduction vise à fournir aux étudiants comprend des compétences technologiques. La traduction automatique, étant l'une des applications les plus importantes des technologies de traduction, attire chaque jour l'attention de plus en plus. Ses usages sont diversifiés et sa valeur marchande augmente. Par conséquent, il est devenu une nécessité d'inclure la traduction automatique et ses applications dans le programme de l'enseignement de la traduction et de former des étudiants à acquérir des compétences dans la traduction automatique. À cette fin, des études pionnières ont été menées dans diverses universités et des moyens pour intégrer la traduction automatique dans l'enseignement de la traduction ont été recherchés. Le sujet de l'enseignement de la traduction automatique dans l'enseignement de la traduction est discuté et diverses idées sont avancées à ce sujet. Deux idées sont généralement admises. La première théorie se concentre sur la logique de travail au lieu d'enseigner les principaux systèmes de la traduction automatique, comme dans les technologies générales de traduction. La seconde conception vise à former des candidats traducteurs dans ce sens, en 
Aslan, E. (2021). La place de la traduction automatique dans l'enseignement de la traduction. Humanias, 9(18), 16-32.

tenant compte des besoins du marché. Quelle que soit l'approche adoptée au point où nous sommes parvenus, les systèmes de la traduction automatique sont désormais devenus un élément indispensable de la formation dans la traduction. Par conséquent, il est d'une grande importance de se concentrer méticuleusement sur ce sujet et de faire de nouvelles recherches pour un enseignement de la traduction automatique plus efficace et efficient. 


\section{Bibliographie}

Akyol, S. \& Fer, S. (2010, Kasım). Sosyal yapılandırmacı öğrenme ortamı tasarımının öğrenenlerin akademik başarılarına ve öğrenmenin kalıcılığına etkisi nedir? International Conference on New Trends in Education and Their Implications. Antalya.

Alkan, S. C. (2013). Lisans düzeyinde çeviri eğitiminde teknoloji eğitiminin yeri. İstanbul Üniversitesi Çeviribilim Dergisi, (7), 127-147.

Austermuehl, F. (2013). Future (and not-so-future) trends in the teaching of translation technology. Tradumàtica, 11(1), 326-337.

Balkul, H.İ. (2015). Türkiye'de akademik çeviri eğitiminde çeviri teknolojilerinin yerinin sorgulanması: müfredat analizi ve ögretim elemanlarının konuya ilişkin görüşleri üzerinden bir inceleme (Yayımlanmamış doktora tezi). Sakarya Üniversitesi, Sakarya.

Bell, R.T. (1991). Translation and translating. Theory and practice. London/New York: Longman.

Bulut, S.Ö. (2019). Integrating machine translation into translator training: towards 'human translator competence? transLogos 2019, 2(2), 1-26.

Durukan, E. \& Çelikay, M. K. (2018). Çeviri edinci kavramına yönelik kapsayıcı bir tanım önerisi. Turkish Studies Language / Literature, 13(12), 161-176.

Ersoy, H. \& Balkul, H. İ. (2012). Teknolojik gelişmelerin çevirmen ve çeviri mesleği açısından olumlu ve olumsuz etkileri: çeviri alanında yeni yaklaşımlar. Akademik Incelemeler Dergisi (Journal of Academic Inquiries), 7(2), 295-307.

Eser, O. (2014). Çeviri eğitiminde edinç kavramının değerlendirilmesi - Türkiye örneği. Uluslararası Sosyal Araştırmalar Dergisi, 7(33), 135-156.

Haldan, A. (2018). Skopos kuramı, çeviri edinci ve çeviribilim öğrencilerinin teknoloji kullanımları üzerine bir değerlendirme. Sinop Üniversitesi Sosyal Bilimler Dergisi, 2(2), 101-112.

Hurtado Albir, A. (1996). La ensenanza de la traduccion directa 'geberal'. Objectivos de aprendizaje y metodologia. In A. Albir Hurtado (Eds.), La ensenanza de la traduccion (pp. 31-55). Castello: Publicacions de la Universitat Jaume I.

Kautz, U. (2000). Handbuch didaktik des übersetzens und dolmetschens. München: Iudicum, Goethe-Institut. 
Aslan, E. (2021). La place de la traduction automatique dans l'enseignement de la traduction. Humanias, 9(18), 16-32.

Killman, J.A. (2015). Introducing machine translation in translator training: Comparing "information mining" with post-editing. Entreculturas. Revista de traducción y comunicación intercultural, (7-8), 179-193.

Kiraly, D. (2014). A social constructivist approach to translator education: empowerment from theory to practice. New York: Routledge.

Koponen, M. (2015). How to teach machine translation post-editing? Experiences from a post-editing course.

https://www.researchgate.net/publication/299346656_How_to_teach_machine_transla tion_post-editing_Experiences_from_a_post-editing_course

Negri, M., Turchi, M., Bertoldi, N. \& Federico, M. (2017). Online neural automatic postediting for neural machine translation. http://ceur-ws.org/Vol-2253/paper63.pdf

Pym, A. (2003). Redefining translation competence in an electronic age. In defence of a minimalist approach. Meta, 48(4), 481-497.

Pym, A. (2013). Translation skill-sets in a machine-translation age. Meta: Journal des traducteurs Translators' Journal, 58(3), 487-503.

Rossi, C. (2017). Introducing statistica machine translation in translator training: from uses and perceptions to course design and back again. Revista Tradumàtica. Tecnologies de la Traducció, 15, 48-62.

Statista (2019). Machine translation market size worldwide, 2016 to 2024. https://www.statista.com/statistics/748358/worldwide-machine-translation-market-size

Şahin, M. (2014). Using MT postediting for translator training. Tralogy. http://lodel.irevues.inist.fr/tralogy/index.php?id=255\&format=print

Vu, T.T. \& Haffari, G. (2018, November). Automatic post-editing of machine translation: A neural programmer-interpreter approach. Conference on Empirical Methods in Natural Language Processing, Brussels.

Yazar, U. (2014). Çeviribilim bölümlerinde verilen derslerin, özellikle bilgi teknolojileri derslerinin, çeviri edinci kazanılmasına katkısı. Dil ve Edebiyat Eğitimi Dergisi, 12, $88-99$.

Yuste, E (2001, September). Making MT commonplace in translation training curricula-too many misconceptions, so much potential. Machine Translation Summit VII, Teaching MT Workshop, Santiago de Compostela. 


\title{
The Place of Machine Translation in Teaching Translation
}

\begin{abstract}
The rapid developments in machine translation in recent years have significantly affected the field of translation. The new methods applied have led to significant improvements in the quality of translation. At the same time, machine translation started to be used in different applications and in different ways, especially on social networks. This situation has made machine translation an area in which large companies are focusing and making significant investments, and as a result, it has increased the market share of machine translation in the sector, and it has made it popular by allowing ordinary people to take an interest in machine translation. Despite this, there is discussion as to how these developments can be reflected in the translator training curriculum and in machine translation. This study focuses on the place of machine translation in Teaching Translation and examines how these systems can be reflected in the Teaching Translation curriculum.
\end{abstract}

Keywords: machine translation, teaching translation, translation, translation technologies 